\title{
Troisième contribution à l'étude faunistique des Culicoides du Maroc
}

\author{
par M. KREMER, M. HOMMEL et H. BAILLY-CHOUMARA \\ (collaboration technique J.-C. Delecolle) \\ Institut de Parasitologie, ( $\mathrm{P}^{r}$ J. Callot), Faculté de Médecine, F. 67 - Strasbourg, \\ Institut scientifique chérifien, Rabat, Maroc
}

\begin{abstract}
Résumé
Les auteurs rapportent les premiers résultats d'une étude portant sur 2995 Culicoides capturés au Maroc. Ils signalent la présence de 14 espèces nouvelles pour le Maroc $(C$. catanéi, $C$. fagineus, C. pulicaris, C. subfascipennis, Culicoides du groupe fascipennis, proche de pallidicornis. C. dhzafarovi, C. subravus, C. sejfadinéi, C. stackelbergi, C. dérisor, C. jumineri, C. similis, C. montanus et $C$. marcleti) ce qui porte à 38 le nombre d'espèces actuellement connues au Maroc.
\end{abstract}

\section{Summary}

The autors report here the first results of a study about 2995 Culicoides (Diptera, Ceratopogonidae) caught in Morroco. They show the existence of 14 new species hitherto unknown in Morroco, which now brings the number of known-species in Morroco to 38 .

Cette étude a été réalisée à partir de Cératopogonidés capturés par l'un des auteurs à l'aide de divers pièges lumineux (hublot lampe, drap piège, piège $C D C$, piège Uv) au Maroc au cours de l'année 1969. Une note plus complète avec les détails des différents prélèvements sera publiée ultérieurement. Nous nous contenterons de signaler ici, les 
espèces non encore connues au Maroc et leurs caractères particuliers, ainsi que des nouvelles localisations pour des espèces déjà signalées.

1. C. catanei Clastrier a été trouvé à Oued Cherrat et Rabat.

2. C. fagineus Edwards, trouvé à Khemisset, Marrakech, Rabat et Sidi Bettache.

3. C. pulicaris Linné, trouvé à Khenifra, Oulmès, Torkoz, Lalla Outka et dans le haut Atlas.

4. C. subfascipennis Kieffer, trouvé à Khenifra, Lalla Outka et Oued Cherrat.

5. C. puncticollis Becker, déjà signalé, a été retrouvé dans les mêmes endroits et dans certains gîtes nouveaux (Fez, Khenitra, Khemisset, Sidi Bettache). Nous sommes actuellement d'avis que les exemplaires que nous avons appelés $C$. riethi précédemment, sont en fait des $C$. puncticollis.

6. C. dérisor Callot et Krémer: un mâle de cette espèce a été trouvé à Rabat et présente tous les caractères de l'espèce, en particulier les paramères typiques à extrémité bifurquée.

7. C. sylvarum Callot et Krémer: un mâle a été trouvé Khenifra.

8. C. scoticus Downes et Kettle: des mâles ont été trouvés à Khenifra et Ourika.

9. C. dhzafarovi Remm : un mâle d'aspect typique a été retrouvé à Oued Cherrat. Dans ce même prélèvement nous avons trouvé cinq femelles ressemblant par beaucoup de points à $C$. dhzafarovi (en particulier par les petites spermathèques rondes très caractéristique 3) mais présentant néanmoins certaines divergences, notamment dans la répartition des sensilles. Alors que la répartition la plus typique est : sensilles présents en 3, 9 , $11,12,13,14$ avec parfois des sensilles en 7, la répartition rencontrée chez les exemplaires marocains est $3,10,11,12,13,14$ avec parfois des sensilles en 6 ou en 9. Par ailleurs les épines tibiales sont toutes sensiblement plus longues. Nous pensons néanmoins que ces légères différences ne permettent pas actuellement de séparer ces espèces et qu'il convient de les appeler $C$. dhzafarovi en signalant cette possibilité de répartition sensillaire.

10. C. stackelbergi Dhzafarov: deux femelles de cette espèce ont été trouvées à Torkoz. Ces femelles présentent les caractères suivants : ailes sans taches de $1150 \mathrm{mi}-$ crons de long, 555 microns de large et 650 microns de longueur costale. Les antennes mesurent 465 microns; l'indice antennaire moyen est de 0,94 . Les sensilles sont présents sur tous les articles courts et absents sur les articles longs. Deux spermathèques de 46 microns. Le palpe mesure 190 microns et le rapport du $3^{\circ}$ article sur les deux précédents est de 1,5. Le palpe présente une fossette sensorielle, de taille moyenne, conique, à ouverture plus petite que la base, telle que la dessine Dhzafarov, et non comme celle de C. pallidus dessinée par Khalaf. Nous pensons que contrairement à l'opinion de Gutsévitch, ces deux espèces ( $C$. pallidus et $C$. stackelbergi) ne sont pas synonymes.

11. Culicoides du groupe Fascipennis. Nous avons rencontré au Maroc trois exemplaires mâles ressemblant à $C$. odiatus tel que le dessine Remm (1967). Par ailleurs, nous avons trouvé dans le même gîte (Torkoz) 13 exemplaires femelles très voisines de C. pa!lidicornis dont les caractères sont les suivants : ailes de 940 microns de long, 454 
microns de large. Antennes de 463 microns de long, avec un indice antennaire de 1,06. Présence de sensilles uniquement sur les articles longs. Longueur du palpe : 163 microns et rapport moyen de 0,90 . Il s'agit par conséquent d'une petite espèce du groupe, n'entrant pas dans le diagramme de Campbell, Pelham Clinton (1959, p. 191, fig. 10).

12. C. agathensis Callot, Kremer et Rioux: de nombreux exemplaires de cette espèce ont été trouvés à Larache, Oulmès, Sidi Bettache, Tétouan. Parmi ces exemplaires nous avons trouvé trois mâles présentant une anomalie assez intéressante sous forme de spicules au niveau du corps de l'aedeagus (voir dessin $n^{\circ} 1$ ).

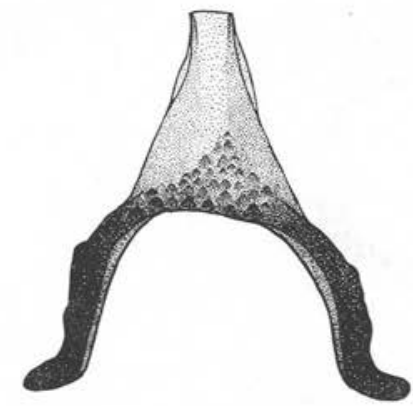

FIG. 1. - C. agathensis: aedeagus

13. C. pseudopallidus Khalaf : 16 femelles de cette espèce, déjà signalée au Maroc, ont été trouvées à Marrakech, Rhafsai. Il faut signaler que les femelles de cette espèce sont très proches des femelles de $C$. hétéroclitus Callot et Krémer, et en l'absence de mâles la diagnose peut être difficile et celle que nous avons portée est peut-être fausse.

14. C. picturatus Callot et Krémer: cette espèce, déjà signalée au Maroc, a été trouvée à Oulmès et Boulesham. Les exemplaires mâles et l'un des exemplaires femelles que nous avons trouvés présentent des taches alaires plus importantes que dans la description originale, avec notamment la présence d'une petite tache à la base de la cellule $\mathrm{mI}$, et une deuxième cellule radiale légèrement entamée par la tache claire. Par ailleurs, les fossettes sensorielles du palpe des femelles sont tantôt uniques, comme dans la description, tantôt multiples (habituellement une grande et une petite, accolées).

15. C. similis Carter, Ingram et Macfie : un exemplaire femelle caractéristique par l'aspect des ailes a été trouvé à Torkoz.

16. C. imicola Kieffer : cette espèce avait déjà été signalée au Maroc sous le nom de $C$. pallidipennis. Elle a été retrouvée à Fez, Rhafsai et Torkoz.

17. C. mon:anus Schakirzyanova (1962): Espèce du groupe obsoletus par la disposition de ses taches alaires, la répartition des sensilles sur les 5 derniers articles antennaires, par la présence d'yeux soudés, l'aspect des spermathèques chez la femelle, et par l'aspect général de l'hypopygium chez le mâlc. Cette espèce se distingue d'obsoletus surtout par l'aspect des antennes et du palpe de la femelle. les antennes présentent en effet des soies transparentes épaiszes et trapues très bien représentées par Gutsévitch (1966). 


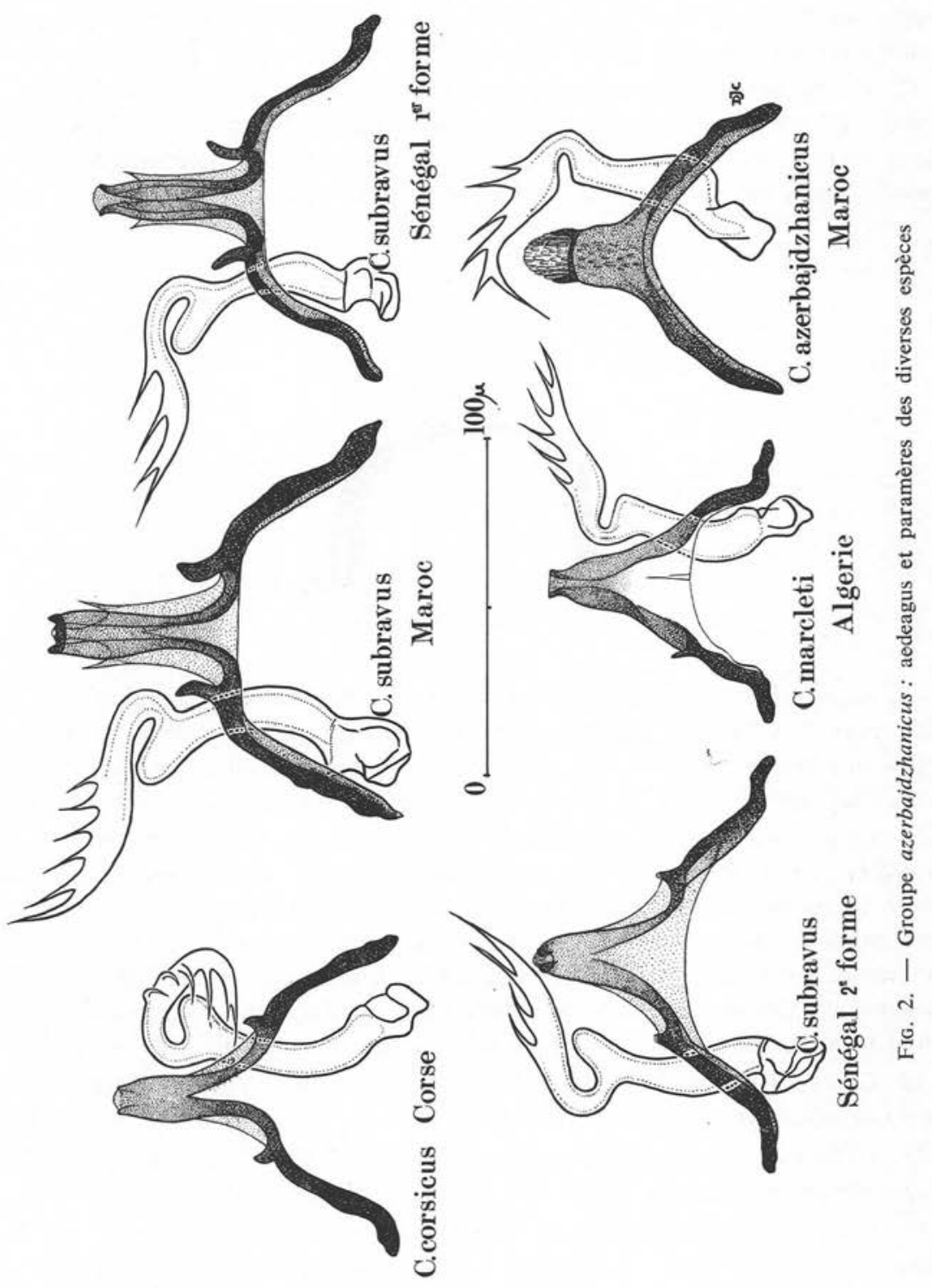


Le palpe moyennement renflé a un appendice cylindrique allongé au fond duquel se trouve la fossette sensorielle. Cet aspect de la fossette sensorielle est tout à fait caractéristique.

Nous avons trouvé 10 exemplaires femelles de cette espèce au Maroc (Marrakech, Torkoz et Oued Cherrat). Ces femelles présentent les caractères suivants: longueur moyenne des ailes $0,997 \mathrm{~mm}(0,865-1,154)$, largeur moyenne $0,481 \mathrm{~mm}$, costa moyenne $0,612 \mathrm{~mm}$, nervure cubitale $0,498 \mathrm{~mm}$. Yeux soudés, non pubescent. Deux spermathèques subégales ( 44 et 42 microns). L'indice antennaire moyen est de 1,07 (extrêmes 0,90 et 1,13 ). La longueur moyenne de l'antenne est de $0,468 \mathrm{~mm}$. La répartition des sensilles est constante : présence de sensilles en $3,11,12,13,14,15$. Le rapport des articles du palpe ( $3^{\circ}$ article sur les deux premiers) est voisin de 1 . Il y a 5 épines tibiales. Pour le mâle de cette espèce nous n'avons malheurreusement pas trouvé de caractères différentiels suffisants qui permettent de le différencier de $C$. obsoletus.

18. Culicoides du groupe azerbajdzhanicus. Il est nézessaire, à notre avis, de rassembler au sein d'un groupe, $C$. azerbajdzhanicus Dhzafarov, C. marcleti Callot et Kremer, $C$. subravus Cornet et $C$. corsicus Kremer, Leberre et Beaucournu-Saguez.

Ce groupe présente un certain nombre de caractères communs: ailes sans taches, hypopygium du mâle caractéristique de type accraensis, répartition sensillaire des femelles (présence de sensilles sur les articles courts uniquement), aspect du palpe, présence de deux spermathèques égales, yeux séparés.

Nous avons exclu de ce groupe $C$. ravus de De Meillon dont le mâle n'est pas attribué avec certitude et dont les femelles présentent des yeux soudés. (Cf. remarque 1).

Ces 4 espèces sont extrêmement voisines et seuls des caractères fins permettent de les distinguer. Nous avons groupé les caractères pouvant aider le diagnostic dans un tableau.

Les différents types d'aedaegus et de paramères ont été schématisés (schéma 2) en respectant les différences de taille.

Au niveau de l'abdomen des femelles existe un petit anneau sclérifié réunissant les canaux en provenance des spermathèques. Nous avons utilisé la forme de cet anneau comme caractère distinctif. On peut en effet rencontrer 2 formes nettement différentes : l'une conique et l'autre en «champignon» (voir dessin $\mathrm{n}^{\circ} 3$ ). Au Maroc nous avons trouvé $C$. subravus Cornet (Marrakech, Torkoz, donc dans la zone présaharienne) : 41 femelles et 8 mâles.

FIG. 3. - Anneau sclérifié de type conique (gauche) et de type en «champignon» (droite) des femelles du groupe azerbajdzhanicus
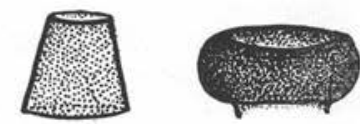

Ces exemplaires marocains semblent être une race géographique de subravus car, bien qu'étant morphologiquement semblables à subravus, ils sont sensiblement plus grands.

C. marcleti : 16 femelles ont été rencontrées à Rhafsai.

C. azerbajdzhanicus Dhzafarov, sensu Gutsévitch : 30 femelles et 1 mâle ont été 
trouvés à Marrakech, Torkoz, Khenifra et Rhafsai. Ces exemplaires sont tout à fait conformes aux caractères indiqués par Gutsévitch (1966) et à des exemplaires qu'il nous a confiés. Mais nous pensons qu'en fait nos exemplaires et ceux de Gutsévitch correspondent à une espèce différente de celle décrite par Dhzafarov (par la taille, l'aspect du palpe, les spicules sur la membrane basale). Nous continuons néanmoins à l'appeler provisoirement $C$. azerbajdzhanicus.

19. C. badooshensis Khalaf: Cette espèce déjà connue au Maroc (Marrakech) a été retrouvée à $\mathrm{Fez}$, Boulesham, Sidi Jahia et Oued Cherrat.

20. C. jumineri Callot et Kremer: Nous avons trouvé 4 mâles et 27 femelles de cette espèce à Torkoz, Fez, Oued Cherrat. Ces exemplaires sont tout à fait identiques aux exemplaires tunisiens qui ont servis à la description de l'espèce, sauf en ce qui concerne la répartition des taches alaires. En effet, chez ces exemplaires marocains il n'y a pas de taches dans les cellules distales de l'aile. Nous pensons que ce seul caractère dif-

Caractères des espèces du groupe Azerbajdzhanicus

\begin{tabular}{|c|c|c|c|c|c|c|}
\hline & Corsicus & $\begin{array}{c}\text { Subravus (1) } \\
\text { origine } \\
\text { marocaine }\end{array}$ & $\begin{array}{c}\text { Subravus (1) } \\
\text { origine } \\
\text { sénégalaise }\end{array}$ & Marcleti & $\begin{array}{c}\text { Azer- } \\
\text { bajdzhanicus } \\
\text { sensu } \\
\text { Gutsévitch }\end{array}$ & \\
\hline $\begin{array}{l}\text { Longueur de } \\
\text { l'antenne ... } \\
\text { Indice anten- } \\
\text { naire ...... } \\
\text { Palpe L .... } \\
\text { Rapport du } \\
\text { palpe } 3 / 1+2 \\
\text { Aile L ..... } \\
\text { L/costa .... } \\
\text { Costa/cub ... } \\
\text { Ann. sclérifié } \\
\text { Spermath. .. }\end{array}$ & $\begin{array}{c}1,47-1,60 \\
163-172 \\
\\
1,20-1,43 \\
1050-1 \quad 178 \\
1,66-1,72 \\
1,31-1,35 \\
\text { champignon } \\
56,7-69,3\end{array}$ & $\begin{array}{c}1,14-1,29 \\
165-206 \\
\\
1,51-1,92 \\
1013-1274 \\
1,61-1,80 \\
1,27-1,46 \\
\text { champignon } \\
49-73,5\end{array}$ & $\begin{array}{c}361-392 \\
1,03-1,24 \\
133-150 \\
\\
1,30-1,60 \\
772-841 \\
1,71-1,79 \\
1,21-1,30 \\
\text { champignon } \\
56-73\end{array}$ & $\begin{array}{c}273-348 \\
1-1,2 \\
109-136 \\
\\
0,95-1,33 \\
616-800 \\
1,92-2,09 \\
1,04-1,20 \\
\text { conique } \\
31-60\end{array}$ & $\begin{array}{c}327-424 \\
0,96-1,25 \\
113-152 \\
\\
1,04-1,61 \\
732-932 \\
1,73-1,95 \\
1,05-1,25 \\
\text { conique } \\
38-67\end{array}$ & ๕ٍ \\
\hline $\begin{array}{l}\text { Aile } \mathrm{L} \ldots . . . \\
\mathrm{L} / \text { costa } \ldots . \\
\text { Costa/cub ... } \\
\text { Antenne L . } \\
\text { Indice anten- } \\
\text { naire } \ldots . . . \\
\text { Thorax ...... } \\
2^{e} \text { cellule ra- } \\
\text { diale sombre }\end{array}$ & $\begin{array}{c}945-959 \\
1,97 \\
1,02 \\
573-582 \\
0,73-0,77 \\
\text { taché } \\
0\end{array}$ & $\begin{array}{l}865-999 \\
1,86-2 \\
1,04-1,25 \\
460-524\end{array}$ & $\begin{array}{c}637-739 \\
2,02-2,13 \\
0,95-1,05 \\
415-459\end{array}$ & $\begin{array}{c}0,60 \\
\text { non taché } \\
+\end{array}$ & $\begin{array}{c}569-680 \\
2,15-2,23 \\
0,86-0,94 \\
457 \\
0,60 \\
\text { taché } \\
0\end{array}$ & ๕๕్ \\
\hline
\end{tabular}

(1) Selon M. Cornet (communication orale) C. subravus pourrait être synonyme de $C$. fuscicaudae Macfie (1947). C. fuscicaudae lui-même pourrait être mis en synonymie avec $C$. ravus De Meillon (1936), qui, en effet, contrairement à la description originale présenterait des yeux séparés chez la femelle sur les exemples déposés en Afrique du Sud. 
férentiel ne suffit pas pour considérer ces exemplaires comme une espèce nouvelle. Cette anomalie alaire rapproche $C$. jumineri d'autres espèces ayant des taches alaires voisines et dont le mâle présente un hypopygium de type cubitalis.

La diagnose est particulièrement difficile (surtout pour les femelles) avec $C$. vidourlensis et $C$. badooshensis. Ceci nous a amené à regrouper les caractères différentiels dans un tableau.

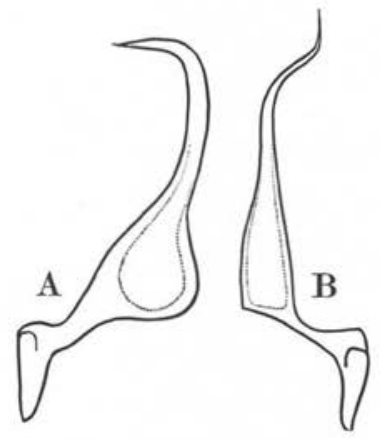

Fig. 4. - C. badooshensis (A),

C. jumineri, et $C$. vidourlensis

(B) : paramères

21. C. sejfadinei Dhzafarov (1958) est une espèce à ailes sans taches et dont la femelle présente trois spermathèques d'aspect typique. Par ces caractères, cette espèce se rapproche de $C$. saevus Kieffer, $C$. ibéricus Dhzafarov et $C$. tauricus Gutsévitch.

Nous avons trouvé au Maroc (Torkoz) 25 femelles de cette espèce. Ces femelles présentent les caractères suivants: ailes sans taches: longueur moyenne $1,315 \mathrm{~mm}$ $(1,19-1,48)$, largeur $0,601 \mathrm{~mm}$, costa moyenne $0,797 \mathrm{~mm}$, nervure cubitale $0,556 \mathrm{~mm}$; Indice antennaire moyen 0,99 (extrêmes $0,94-1,06$ ) ; Longueur moyenne de l'antenne 0,492 $\mathrm{mm}$; Sensilles antennaires présents constamment en 3, 6, 7, 8, 9, 10, parfois en 5 (cette répartition sensillaire est commune au groupe). Les yeux des femelles sont séparés, non pubescents. Il y a 5 épines tibiales. Les articles du palpe $(3 / 2+1)$ sont dans le rapport voisin de 1 .

\begin{tabular}{|c|c|c|c|}
\hline & C. jumineri & C. vidourlensis & C. badooshensis \\
\hline $\begin{array}{l}\text { Sensilles sur le } 15^{\circ} \text { article an- } \\
\text { tennaire (mâle et femelle) } \ldots\end{array}$ & + & + & 一 \\
\hline Indice antennaire (femelle) $\ldots$ & $1,1-1,3$ & $1,4-1,6$ & $1-1,03$ \\
\hline Taches à l'extrémité de l'aile & + à - & - & - \\
\hline Membrane basale spiculée .. & ++ & - & \pm \\
\hline 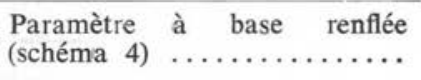 & - & - & + \\
\hline
\end{tabular}


Culicoides du Maroc

\begin{tabular}{|c|c|c|c|c|c|c|c|c|c|}
\hline & 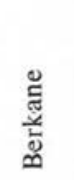 & 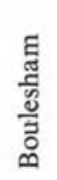 & 先 & 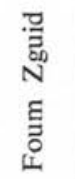 & $\begin{array}{l}\text { 壳 } \\
\text { Ȯ }\end{array}$ & 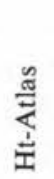 & 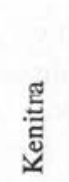 & 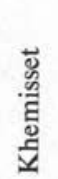 & $\begin{array}{l}\text { 면 } \\
\text { है } \\
\text { है }\end{array}$ \\
\hline Lailae $\quad \ldots \ldots \ldots \ldots \ldots \ldots$ & & & & & & & + & & \\
\hline Halophilus $\quad \ldots \ldots \ldots \ldots$ & & + & + & & & & $(+)$ & & + \\
\hline Circumscriptus $\ldots \ldots \ldots$ & & & + & & $(+)$ & + & $(+)$ & & \\
\hline Punctatus $\quad \ldots . \ldots \ldots \ldots$ & $(+)$ & & & $(+)$ & & & & & \\
\hline Agathensis $\quad \ldots \ldots \ldots \ldots$ & & & & & & & & & \\
\hline Puncticollis $\ldots . . \ldots \ldots \ldots$ & $(+)$ & & + & & & & + & + & \\
\hline Coluzzii ................ & $(+)$ & & + & & & & & + & \\
\hline Pulicaris $\ldots \ldots \ldots \ldots \ldots$ & & & & & & + & & & + \\
\hline Langeroni $\ldots . . . \ldots \ldots \ldots$ & & & & & & & & & \\
\hline Catanei $\ldots \ldots \ldots \ldots \ldots$ & & & & & & & & & \\
\hline Parroti $\quad \ldots \ldots \ldots \ldots \ldots$ & & & & & & & & & \\
\hline Longipennis $\quad \ldots \ldots \ldots \ldots$ & $(+)$ & & & & & & & & \\
\hline Similis $\quad \ldots \ldots \ldots \ldots \ldots$ & & & & & & & & & \\
\hline Kingi-Schultzei $\ldots \ldots \ldots$ & & & & $(+)$ & & & & & \\
\hline Subfascipennis $\quad . . \ldots \ldots \ldots$ & & & & & & & & & + \\
\hline Fascipennis groupe $\ldots . .$. & & & & & & & & & \\
\hline Santonicus $\quad \ldots \ldots \ldots \ldots$ & & & & & & & & & \\
\hline Sejfadinei $\quad \ldots . \ldots \ldots \ldots$ & & & & & & & & & \\
\hline Saevus $\quad \ldots \ldots \ldots \ldots \ldots$ & & & + & & & + & & & \\
\hline Maritimus $\quad \ldots \ldots \ldots \ldots$ & & & & & & & & & \\
\hline Odibilis $\ldots \ldots \ldots \ldots \ldots$ & & & & & & & & & \\
\hline Montanus $\ldots \ldots \ldots \ldots$ & & & & & & & & & \\
\hline Azerbadjanicus .......... & & & & & & & & & + \\
\hline Marcleti $\quad \ldots \ldots \ldots \ldots \ldots$ & & & & & & & & & \\
\hline Derisor $\quad \ldots \ldots \ldots \ldots \ldots$ & & & & & & & & & \\
\hline Badooshensis $\quad . . . \ldots \ldots$ & & & + & & & & & & \\
\hline Dhzafarovi $\ldots . . \ldots \ldots \ldots$ & & & & & & & & & \\
\hline Sylvarum $\ldots \ldots \ldots \ldots$. & & & & & & & & & + \\
\hline Scoticus $\ldots \ldots \ldots \ldots \ldots$ & & & & & & & & & + \\
\hline Picturatus $\ldots \ldots \ldots \ldots$ & & + & & & & & & & \\
\hline Stackelbergi $\ldots \ldots \ldots \ldots$ & & & & & & & & & \\
\hline Pseudopallidus $\ldots \ldots \ldots$. & & & & & & & & & \\
\hline Obsoletus $\quad \ldots \ldots \ldots \ldots \ldots$ & & & & & & & & & + \\
\hline Imicola $\ldots . . . \ldots \ldots \ldots$ & & & + & & & & & & \\
\hline Begueti $\quad . . \ldots \ldots \ldots \ldots$ & & & & & & & & & \\
\hline Subravus $\ldots . \ldots \ldots \ldots \ldots$ & & & & & & & & & \\
\hline Fagineus $\ldots . . . \ldots \ldots \ldots$ & & & & & & & & & + \\
\hline Jumineri $\quad \ldots \ldots \ldots \ldots \ldots$ & & & + & & & & & & \\
\hline
\end{tabular}




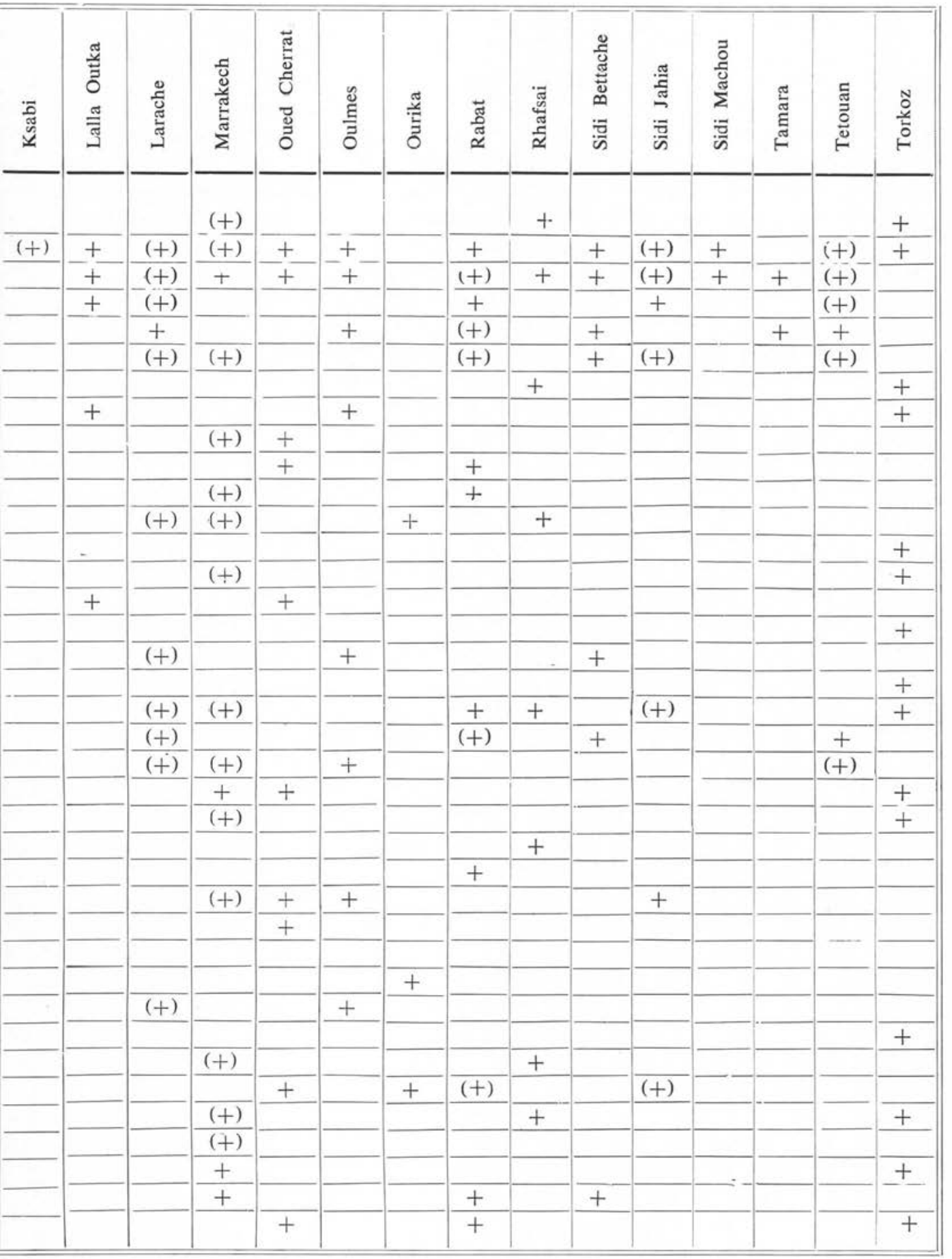

+ : nouvelle localisation.

$(+)$ : localisation déjà signalée. 


\section{Bibliographie}

Bailly-Choumara (H.) et Kremer (M.), 1970. - Deuxième contribution à l'étude des Culicoides du Maroc. Cahiers de l'O.R.S.T.O.M., VIII, 383-391.

Callot (J.), Krémer (M.) et Bailly-Choumara (H.), 1968. - Note faunistique préliminaire sur les Culicoides du Maroc. Gynandromorphisme de C. curcumscriptus parasité par un mermis. Bull. Soc. Path. Exot., 61, 885-889.

Callot (J.), Krémer (M.) et Basset (M.), 1968. - Culicoides marcleti n. sp. et nouvelles localisations de Culicoides de la région méditerranéenne et particulièrement d'Algérie. Bull. Soc. Path. Exot., 61, 271-282.

Callot (J.) et Krémer (M.), 1965. - Sur quelques Diptères nématocères du Var avec la desoription d'espèces nouvelles de Culicoides. Annales de Parasitologie, 40, 329-339.

Callot (J.) et Krémer (M.), 1969. - Description d'un Culicoide nouveau C. jumineri trouvé en Tunisie. Bull. Soc. Path. Exot., 62, 1112-1118.

Campbell (J.-A.) et Pelham-Clinton (E.-C.), 1959-1960. - A taxonomic review of the British species of Culicoides Latreille. Proc. R. Soc. Edinburgh (B), 68, 181-302.

Cornet (M.) et Chateau (R.). - Culicoides de l'Ouest africain ( $2^{\circ}$ note). Espèces apparentées au groupe Similis. Cahiers de l'O.R.S.T.O.M., VIII, 141-173.

Dhzafarov (M.), 1964. - Heleidae du Caucase: morphologie, biologie, et répartition géographique. Rôle pathogène. Moyens de lutte. Faune des genres Culicoides, Leptoconops et Lasiohelea. Akademia Nauk Azerbadjanskoj SSR. Inst. Zoo.

Gutsevitch (A. V.), 1966. - Keys to bloodsucking midges of the genus Culicoides from Middle Asia. Revue d'entomologie d'U.R.S.S., XLV: 658-678.

KhaLAF (K. T.), 1961. - More Culicoides from Irak. Beiträge zur Ent., 11, 450-470.

Kremer (M.), 1965. - Contribution à l'étude du genie Culicoides, Latreilfe particulièrement en France. Lechevallier éd., Paris.

Kremer (M.). - Redescription de C. imicola, C. alticola, C. tropicalis Kieffer sur des exemplaires déterminés par l'auteur et conservés au Museum National d'Histoire Naturelle de Paris. Bulletin du Museum (sous-presse).

Kremer (M.), Leberre (G.), et Beaucournu-Saguez (F.). - Note sur les Culicoides de Corse. Description de C. corsicus n. sp. Ann. Par. Hum. Comp. (sous-presse).

Remm (M.-J.), 1967. - On the fauna of Ceratopogonidae in the Caucasus. Zool. Aladeid Toïd., IV: 3-38. 